\title{
The Influence of Jam Density and Merging Cyclists on the Queue Discharge Rate
}

\author{
M. J. Wierbos $\mathbb{D}$, V. L. Knoop $\mathbb{D}$, B. Goñi-Ros $\mathbb{D}$, and S. P. Hoogendoorn \\ Transport \& Planning, Delft University of Technology, Delft, Netherlands \\ Correspondence should be addressed to M. J. Wierbos; m.j.wierbos@tudelft.nl
}

Received 22 January 2020; Revised 24 July 2020; Accepted 31 July 2020; Published 28 August 2020

Academic Editor: Shamsunnahar Yasmin

Copyright (C) 2020 M. J. Wierbos et al. This is an open access article distributed under the Creative Commons Attribution License, which permits unrestricted use, distribution, and reproduction in any medium, provided the original work is properly cited.

\begin{abstract}
An increasing number of people use the bicycle for urban trips resulting in local congestion at intersections, especially during peak hours. Understanding the queue dynamics is key to find the correct measures that can reduce the delays for cyclists without affecting other traffic modes. To this end, the discharge process of bicycle queues is studied, focusing on the impact of jam density on the queue discharge rate and how this process is affected by cyclists that merge into the queue during the discharge phase. The impact of merging cyclists is captured by a newly introduced bicycle equivalent (BE) value. This direction-specific BE value is used to convert a merging cyclist into a cyclist that is waiting in the original queue. Results show that the queue discharge rate increases with increasing density of the queue. Furthermore, cyclists that merge by overtaking contribute to the queue discharge rate, while cyclists who merge from a perpendicular direction hinder the discharge process, thereby decreasing the bicycle flow at the intersection. The insights can be used to develop measures which minimise delay at intersections and to design efficient infrastructure for bicyclists.
\end{abstract}

\section{Introduction}

Bicycles are gaining popularity as a mode of transport, especially in Western European countries and regions with a positive policy towards cycling $[1,2]$. Around $27 \%$ of all daily trips in the Netherlands were made by bicycles between 1995 and 2005 [3], and this increased to 32\% in 2016 [4]. Besides this development in usage, also the distance travelled per trip expanded, leading to more cyclists on the roads [5]. The increased bicycle traffic has led to local congestion at intersections during peak hours, even though the Dutch cycling infrastructure is well developed. Little is known about the dynamics of bicycle flow, and more research is required to identify measures that can help prevent future bicycle congestion.

This study focuses on the queue dynamics at a controlled intersection to gain insight into the factors that influence the queue discharge rate, e.g., jam density. Cyclists have freedom in choosing their position on the road, especially when joining a queue. Depending on how compact the people position themselves, a bicycle queue can have different densities. When moving, the density relates to flow according to the fundamental diagram in traffic flow theory. If and how such a relation holds for jam density and queue outflow is open to question.

Furthermore, it is unclear how so-called "merging cyclists" affect the outflow (Figure 1). These merging cyclists are additional cyclists that merge into the flow during the queue discharge process, for example, by overtaking the queue from behind or joining the queue from the side. The influence of merging cyclists is linked to the layout of the intersection and the number of approach directions. If there are open spaces in the queue to absorb the additional cyclists, the merging behaviour might have a positive effect on the overall flow. However, if the merging behaviour obstructs the queue discharge process, the overall capacity might reduce. This leads to the following question: What is the impact of jam density and merging cyclists on the queue discharge rate at an intersection?

This study aims to quantify the influence of jam density and merging cyclists on the queue discharge process. We do so by analysing the queuing events of a real-life intersection 


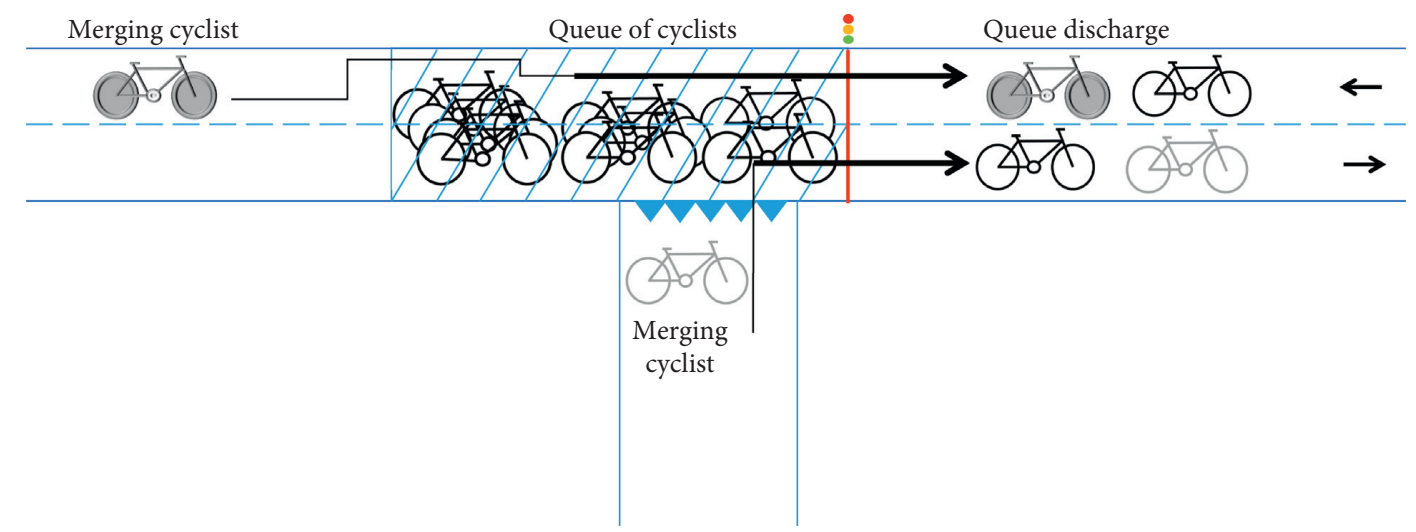

FIGURE 1: Sketch of a queue discharge process: the original queue is indicated by the black cyclists in the hatched area, and the black arrows indicate merging cyclists who skip part of the queue and join the discharge before the queue is cleared.

with multiple approach directions. The results of this case study are then qualitatively validated in a small-scale controlled experiment. This setting allows us to keep the group composition constant, which reduces the intrinsic variability of the observed discharge times. The results are expected to differ quantitatively from the real-life case study due to different infrastructural setting and group composition. However, they will suffice to validate the sign of the influence of jam density on the queue discharge time and discharge rate. The acquired knowledge can be used to evaluate existing infrastructure with respect to cyclist delay or to design new intersections where the delay for cyclists is reduced. The results will be of interest especially for countries with high bicycle volumes, such as the Netherlands or Denmark.

The paper is build up as follows: Section 2 provides background information on bicycle traffic flow and equivalent units, Section 3 describes the research methodology, and Section 4 describes the case study which is used to analyse the queue discharge process. Section 5 shows the results of the analysis, and Section 6 gives details of the experiment that was used to validate the case study results. The paper ends with a discussion of the results in Section 7 and conclusions in Section 8.

\section{Related Work}

Cyclists have the freedom to choose their lateral position on the road because a bicycle lane is generally wider than the bicycle itself. This enables cyclists to overtake other people or to cycle in pairs within one lane $[6,7]$. At intersections, this freedom results in queues that are less organised than the queues in motorised traffic. A queue of motorised vehicles is structured in lanes, whereas cyclists in a queue are grouped closely together and form a cluster pattern [8]. How this cluster pattern effects the queue discharge process is yet unclear.

The flow characteristics of the bicycle and motorised traffic are comparable under certain conditions [9]. Similar concepts such as speed, flow, and density relations can be used to describe the flow on a macroscopic level [10]. Typical characteristics are capacity, critical density, and jam density, among others. Only a few values have been reported on jam density, all varying around $0.6 \mathrm{bic} / \mathrm{m}^{2}[9,11,12]$. The reported maximum flow rate, or capacity, shows more variation. The highest number has been reported by Navin [9] who performed experiments in 1974 in Vancouver (Canada) and compared the findings with data from real-life situations, resulting in an estimated capacity of about $4,000 \mathrm{bic} / \mathrm{h} /$ $\mathrm{m}$ for a $2.5 \mathrm{~m}$ wide cycle path. Botma and Papendrecht [6] studied bicycle data in the Netherlands, estimating a capacity between 2,600 and $3,600 \mathrm{bic} / \mathrm{h} / \mathrm{m}$ for a $2.5 \mathrm{~m}$ wide cycle path. Hoogendoorn and Daamen [7] reported the lowest capacity value of $1,531 \mathrm{bic} / \mathrm{h} / \mathrm{m}$ based on a new bicycle headway model with the remark that this value might be an underestimation of the actual capacity because the cyclists did not use the full width of the $3 \mathrm{~m}$-wide path. Li et al. [13] looked into mixed bicycle traffic consisting of around $80 \%$ electric bicycles and reported a capacity of around 3,300 bic/h/m. An explanation for the wide variation could be a different definition for the maximum flow rate, namely, saturation flow and capacity.

The saturation flow is the maximum number of cyclists that pass the stop line of an intersection within one unit of time, whereas the capacity takes into account the signal time, resulting in a lower value than saturation flow [14]. Seriani et al. [15] analysed the saturation flow at Tavistock Square (London, UK) and Pocuro (Santiago de Chile) and found that the saturation flow at Tavistock Square depended on the time of day; a maximum of around $4,300 \mathrm{bic} / \mathrm{h} / \mathrm{m}$ was observed in the morning peak hour, while the results were $25 \%$ lower for the afternoon peak hour. The saturation flow showed a linear dependency on lane width at Pocuro, ranging between $2,000 \mathrm{bic} / \mathrm{h} / \mathrm{m}$ on a $1 \mathrm{~m}$ lane and $2,350 \mathrm{bic} / \mathrm{h} / \mathrm{m}$ on a $2 \mathrm{~m}$ lane. Jin et al. [16] also found that the width of a cycle path did not significantly influence the capacity per meter, implying that the total capacity of a cycle path scales linearly to the width of the cycle path. The time interval used to calculate capacity was found to influence the results significantly and with a linear downward trend; the estimated capacity decreased when a larger time interval was used for the estimation. Besides path width and measuring interval, also the configuration of the cyclists in the queue might be of influence to the capacity. 
Bicycle queue formation has been studied by Cao et al. [17], which resulted in two models to describe the relation between queue length, jam density, and density distribution. It was observed that initially, only a portion of the lane width was used for the queue, but when a critical queue length was reached, the additional cyclists would overtake the queue and start to fill the gaps. This process indicates that queues of similar lengths can have different average queue densities. How these merging manoeuvres such as overtaking influences the queue discharge process, however, has not yet been studied.

The discharge process of a bicycle queue has been studied by Goñi-Ros et al. [12], specifically looking at macroscopic characteristics in queuing events without merging cyclists. They found that a higher jam density results in higher discharge flow and shockwave speed. Furthermore, they observed a wide variation between queuing events, which highlights the stochastic nature of bicycle traffic flow. This finding is also stressed in Yuan et al.'s study [18]. who estimated the bicycle flow capacity of an intersection based on the saturation headway and the number of virtual sublanes. The observed number of sublanes was less than the theoretical number, leading to a decrease in the saturation rate of $27-43 \%$. The high variety in reported values for capacity and saturation flow might also be explained by several factors such as infrastructure design, weather conditions, and heterogeneity in the bicycle or bicyclist type. Although it might be interesting to look into these factors, it is out of scope here. We focus on the influence of jam density on the flow rate and aim to capture the influence of merging cyclists on equivalent units.

In motorised traffic, equivalent units are used to account for heterogeneity. The traffic flow at an intersection with multiple approach directions can be considered as heterogeneous since the overall flow consists of multiple subflows with different characteristics. This heterogeneity makes it difficult to compare flow characteristics such as the queue discharge rate. The comparison is made easier when the impact of different factors is described on the same reference unit. The most common reference is the passenger car equivalent (PCE), which was first introduced by the 1965 Highway Capacity Manual [19]. Each vehicle type has a specific correction factor (PCE value) to convert a mixed traffic stream into a uniform flow of PCEs.

Alternative reference modes have been proposed as well to retrieve a homogeneous description of heterogeneous flow. Cao and Sano [20] proposed a motorcycle equivalent (ME) to translate mixed traffic conditions in Hanoi, Vietnam, into a unified motorcycle flow. A mixed flow of bicycles and mopeds was normalised in bicycle equivalent (BE) units by Chen et al. [21]. It was found that mopeds have a $\mathrm{BE}$ value larger than one under high-density conditions and a $\mathrm{BE}$ value smaller than one under low-density conditions. Other research studies focused on retrieving $\mathrm{BE}$ values for different infrastructures and varying mixing ratios for motorised and nonmotorised cyclists. The BE value for mopeds was found to depend on lane width, slope of the road, density, and moped percentage [22]. The impact of electrical bicycles has been captured in a fixed bicycle equivalent value of 0.666 [16] based on observations in free flow, stable, and restricted flow conditions. Equivalent units have not been used before to describe bicycle flow at an intersection with multiple approach directions. We apply this concept to capture the influence of merging cyclists on the queue discharge rate.

\section{Methodology}

Section 3.1 describes the method used to find the influence of jam density on the discharge time and queue discharge rate. Section 3.2 describes the method to include the effect of merging cyclists and introduces the bicycle equivalent unit.

3.1. Analysis of the Influence of Jam Density on the Queue Discharge Process. The influence of the jam density on the queue discharge process is captured via the queue discharge time and discharge rate. The method to determine these variables is discussed first followed by the method to determine their relation.

The density of a queue depends on its dimensions and the number of cyclists in it. For a queue of fixed length, a waiting area can be defined as the length multiplied by the width of the cycle path. The jam density is then calculated by the number of cyclists in the waiting area $\left(N_{q}\right)$ divided by the fixed dimensions of the waiting area $(A)$ :

$$
k_{j}=\frac{N_{q}}{A} .
$$

The duration of the queue discharge process is the discharge time, which is the time interval between the first and the last cyclist in the queue to exit the waiting area: $T_{\text {dis }}=T_{\text {last }}-T_{\text {first }}$. The queue discharge rate $\left(q_{\text {dis }}\right)$ is the number of cyclists $(N)$ that pass the exit line of the waiting area during the discharge time:

$$
q_{\text {dis }}=\frac{N}{T_{\text {dis }}} .
$$

Here, $N$ consists of the cyclists in the queue and the additional cyclists that merge into the queue during the discharge process. If the merging cyclists influence the queue discharge process, both the discharge time and the queue discharge rate will be affected.

Since the cumulative flow $N$ is less volatile than the flow, we choose to show the effects of jam density in this cumulative flow plane. If the queue discharge rate was independent of queue density, the total discharge time $\left(T_{\text {dis }}\right)$ would scale proportionally with the number of cyclists:

$$
T_{\text {dis }}=\frac{N_{q}}{q_{\text {dis }}}=\frac{A k_{j}}{q_{\text {dis }}} .
$$

Figure 2 shows how $T_{\text {dis }}$ would relate to the jam density if this was the case. We will analyse various observed queuing events and show them in this plane. If the queue discharge rate is independent on the jam density, various points will follow one of the coloured lines. If not, the points will cross several lines. We will perform a linear regression of these points of the form 


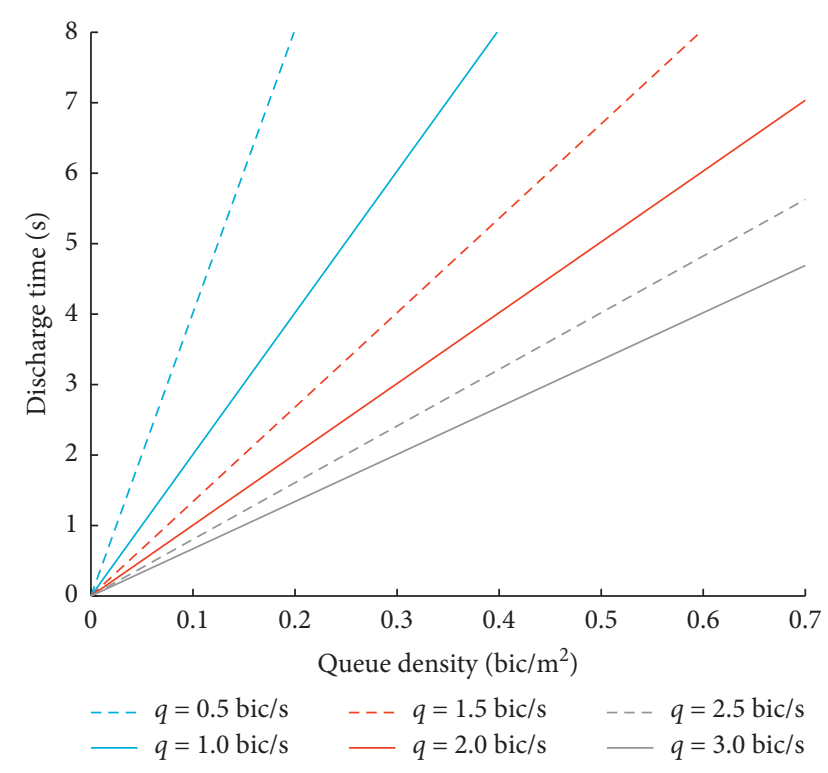

Figure 2: Template to visualize the relation between jam density and queue discharge rate.

$$
T_{\text {dis }}=T_{0}+\beta k_{j}
$$

where $T_{0}$ indicates the offset. We expect a value for $\beta$ larger than 0 . A value for $T_{0}$ equal to zero would indicate that the discharge time is proportional to the jam density $k_{q}($ and $N)$, see equation (3). However, when the $T_{0}$ value is larger than 0 , it would indicate that the term $1 / q_{d}$ decreases with density, and hence, the queue discharge rate increases with the queue density.

3.2. Analysis of the Influence of Merging Cyclists. We will extend the aforementioned analysis to include the effect of merging cyclists on the queue dissipation process also. This impact of merging cyclists is quantified using multiple linear regression analysis. Since the queue discharge rate depends on the discharge time in our definition, see equation (2), we focus the analysis on the queue discharge time and later use the bicycle equivalent unit to quantify the effect of different merging directions. The expression of the multiple linear regression analysis is

$$
T_{\text {dis }}=T_{0}+\mu N_{q}+\sum \alpha_{i} N_{i}
$$

Here, $N_{q}$ is the number of waiting cyclists in the queue, $N_{i}$ is the number of cyclists merging into the queue from direction $i, T_{0}$ is the regression constant, and $\mu$ and $\alpha_{i}$ are the regression coefficients, corresponding to direction $i$. The regression coefficients of different merging directions are tested on a $95 \%$ significance level, and only the directions that lead to the model with the highest adjusted $R$-squared value should be included. The standardised regression coefficients of the final model are used to evaluate and rank the influencing factors for the discharge time. A standardised coefficient is expressed in standard deviations $(\sigma)$ and indicates how many standard deviations the discharge time will change due to the increase of one standard deviation of the independent variable.

Scaling the regression coefficients $\left(\alpha_{i}\right)$ to the main influencing factor $(\mu)$ gives an indication of the impact of different influencing factors. This process of scaling is similar to the concept of passenger car equivalent, which is a measure for the impact factor of different classes in heterogeneous (motorised) traffic [23]. For bicycle traffic, bicycle equivalent $(\mathrm{BE})$ is introduced as a measure for the impact of merging cyclists from direction $i$ relative to the impact of the cyclists on the original queue:

$$
\mathrm{BE}_{i}=\frac{\alpha_{i}}{\mu}
$$

The bicycle equivalent value $\mathrm{BE}_{i}$ represents the impact that a merging cyclist from direction $i$ has on the discharge time. $\mathrm{A} \mathrm{BE}$ value larger than 1 indicates that the merging cyclist has an increased impact on the discharge time, hindering the waiting people more than if the cyclist was standing in the original queue. A BE value smaller than 1 indicates that the merging cyclist has less influence than if they were standing in the waiting area.

Using the $\mathrm{BE}$ values, the total number of cyclists in equation (2) can be recalculated to the reference unit or bicycle equivalent unit (beu). This results in a uniform expression for the queue discharge rate in beu/s,

$$
q_{\mathrm{beu}}=\frac{N_{\mathrm{beu}}}{T_{\mathrm{dis}}}=\frac{N_{q}+\sum\left(B E_{i} N_{i}\right)}{T_{\mathrm{dis}}} .
$$

Merging cyclists with BE value larger than 1 indicates that the overall influence on the outflow rate in bic/s is negative. The negative impact on the discharge time is larger than the positive gain of an additional cyclist in the total cyclist count $N$. After correcting for this impact, their contribution changes to positive in the queue discharge rate expressed in beu/s. Merging cyclists with a BE value smaller than 1 results in a positive influence on the outflow rate when expressed in bic/s, but this influence is reduced when the outflow rate is expressed in beu/s.

\section{Case Study}

The methods described in Section 3 were tested in a case study for which the data were collected in October 2014, see Hoogendoorn and Daamen [7]. The location is the Mekelweg-Jaffalaan-intersection, which is a part of a busy cycling route between the city centre and the university campus in Delft, the Netherlands. Video data were collected over a period of 2 weeks, covering an intersection where bicyclists have priority over cars. The stream of cyclists in the morning peak hour was controlled by human traffic controllers who stopped the stream of cyclists periodically to help car traffic cross the cycle path. An overview of the situation is provided in Figure 3. Due to the proximity to the university, the flow consisted predominantly of students who cycled on a regular basis and might have been rushed to get to their classes in time. 


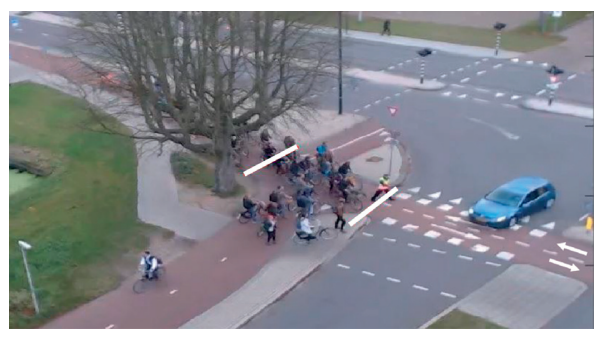

(a)

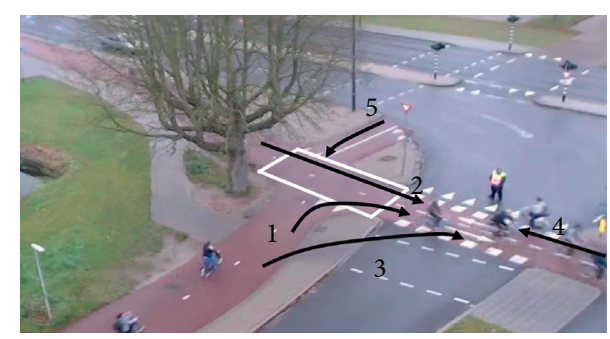

(b)

Figure 3: Example of the video data (a) with the waiting area defined by the white lines. The black arrows indicate different directions in which additional cyclists can influence the queue discharge process (b).

The video was analysed, looking specifically at the moments at which the bicyclists were stopped and released again by the traffic controller. During the blocked periods, a queue of cyclists was formed in front of the intersection using occasionally the full width of the cycle path $(3 \mathrm{~m})$, including the lane for the opposite direction. In the images, a waiting area is defined and drawn by the white lines in Figure $3(\mathrm{~b})$. The size of the waiting area $(A, 6.7 \mathrm{~m} \times 3 \mathrm{~m})$ was chosen such that a clear count of the number of cyclists could be made based on the video images, so its size was limited by the presence of the tree. The events were recorded only when the queue length reached the end of the waiting area or exceeded this length, resulting in a total of 106 queuing events. The number of cyclists within the waiting area was determined manually, resulting in different queue densities depending on how compact the cyclists had positioned themselves. After the traffic controller released the flow, the queue discharge time was determined as the time interval between the first wheel passing the exit line and the second wheel of the last cyclist (of the original queue) passing the exit line. With this method, the initial start-up time of the cyclists was not included in the discharge time. The bicyclists did not have any interaction with vehicles during the discharge time, and interaction with pedestrians was very limited. Based on this knowledge, we assumed in the analysis that the discharge process was only influenced by the cyclist-cyclist interaction.

The observed jam density ranged between 0.2 and $0.6 \mathrm{bic} / \mathrm{m}^{2}$ with a mean of $0.4 \mathrm{bic} / \mathrm{m}^{2}$ and standard deviation of $0.086 \mathrm{bic} / \mathrm{m}^{2}$, see Figure 4 . The time to empty the waiting area ranged from 2 to 7 seconds with a mean of $4.6 \mathrm{~s}$ and standard deviation of $0.92 \mathrm{~s}$. Before the last cyclist of the original queue left the waiting area, additional cyclists could merge into the queue from different directions. The merging cyclists were counted from different directions (see Figure 3(b)), being the cycle path from Jaffalaan which has a 90-degree angle with the waiting area (1), overtaking from behind (2), and a shortcut direction across the side walk (3). Furthermore, the presence of cyclists going in the opposite direction was recorded (4) as well as the cyclists coming from direction (5). Only the queues in the morning peak hour were captured and analysed. At that time of the day, the majority of the flow was directed towards the university building (to the right in Figure 3), and almost no oncoming cyclists were present coming from direction 4 . The number of additional cyclists per discharge period varied between 0 and 6 cyclists for direction 1, 0-4 cyclists for direction 2 , and $0-2$ for direction 3. During the selected queuing events, no bicyclists were observed coming from direction 5 .

\section{Results of the Case Study}

The results of the analysis of the case study data are discussed, starting with the influence of jam density on the discharge time and queue discharge rate followed by a quantification of the influence of merging cyclists, which leads to the queue discharge rate expressed in bicycle equivalent units.

5.1. Influence of Jam Density. The observed queue discharge times are plotted against jam density in Figure 5. The scattered dots show a positive trend, which is captured by the linear fit (black line). The results show that the discharge time increased gradually with jam density, which can be interpreted as a time penalty for density. On the contrary, the queue discharge rate also increases when cyclists stand closer together in the queue, which indicates that the queue dissipation process is more efficient. Linear regression analysis results in the following regression model:

$$
T_{\text {dis }}=2.70+5.05 k_{j} \text {. }
$$

The regression equation is statistically significant $(F(1,104)=30.4, p<.001)$ with an $R$-squared value of 0.226 . This means that the discharge time increases with 5.05 seconds for every bic $/ \mathrm{m}^{2}$ increase in jam density and that the equation explains about $23 \%$ of the variance in the observed discharge time. The model is improved when including the effect of merging cyclists also.

5.2. Influence of Merging Cyclists. Other explanatory factors for the observed variation in the queue discharge time are cyclists that merged into the queue during the discharge process. The multiple regression analysis showed that only the number of waiting cyclists in the queue $\left(N_{q}\right)$, number of merging cyclists from direction $1\left(N_{1}\right)$, and merging cyclists from direction $2\left(N_{2}\right)$ had a significant influence on the discharge time. The influence of merging cyclists from direction $3\left(N_{3}\right)$ and oncoming cyclists $\left(N_{4}\right)$ is not statistically significant and has therefore been excluded from the final 


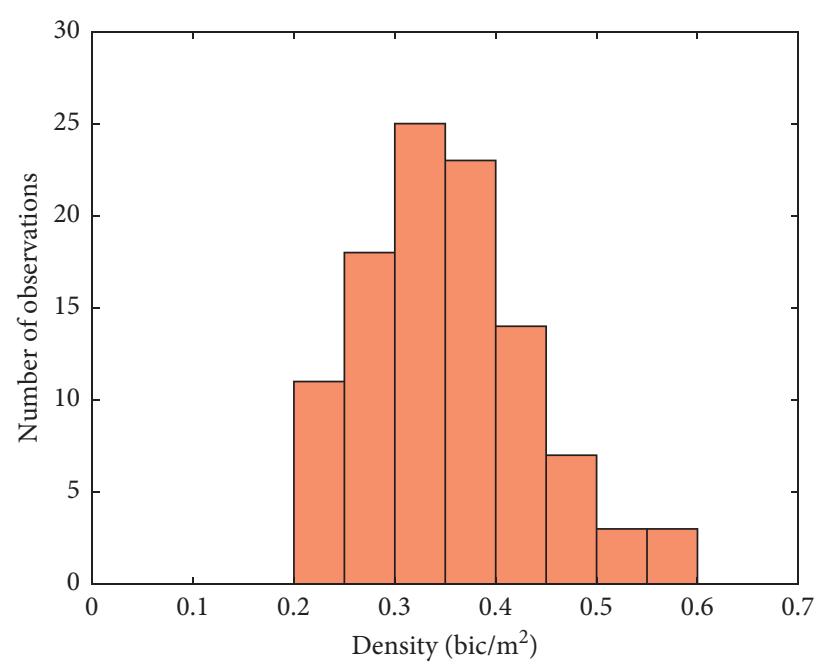

(a)

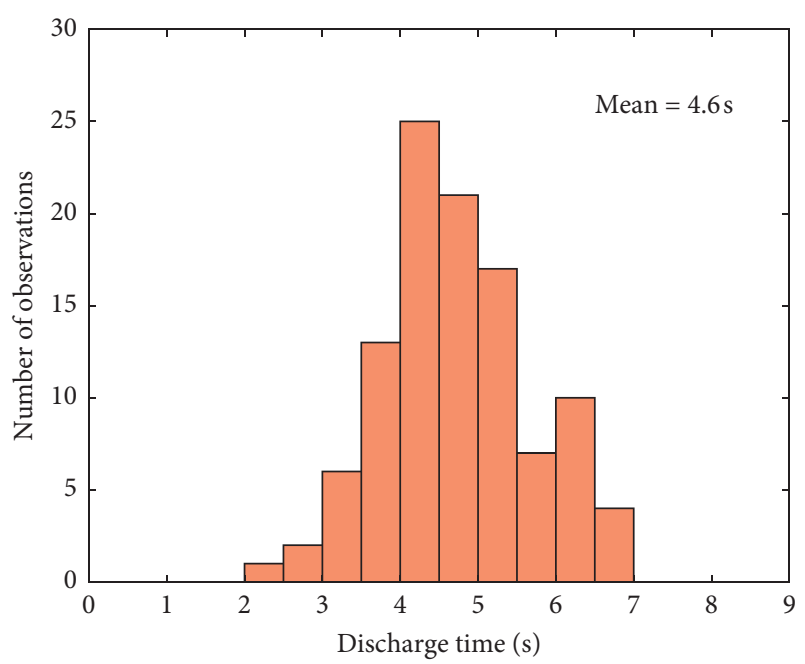

(b)

Figure 4: Frequency distributions.

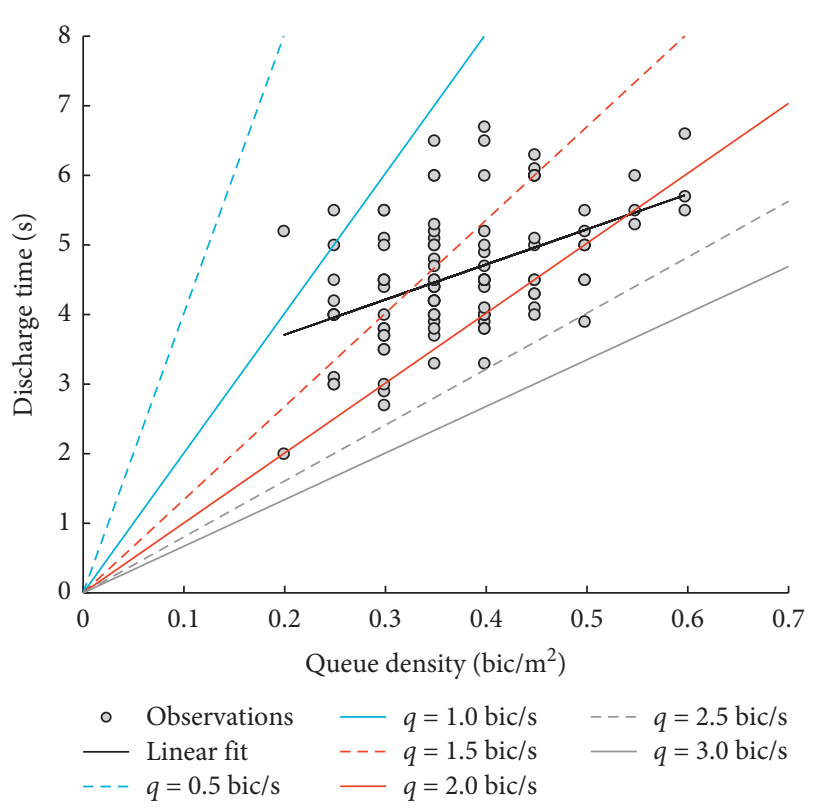

FIGURE 5: Observed discharge time and jam density (dots) and the linear regression fit (black line). The lines starting from the origin indicate different flow rates in bic/s over the full width of the cycle path $(3 \mathrm{~m})$.

regression analysis. Table 1 provides the results of the Pearson correlation. It shows that the correlation between discharge time and both $N_{q}$ and $N_{1}$ is around 0.50 , indicating that these variables had a moderate and positive impact on the discharge time. The correlation between discharge time and merging cyclists from direction 2 is lower, around 0.25 , indicating that $N_{2}$ had a possible positive impact on the discharge time, but the connection was weak. The correlation between $N_{q}$ and $N_{1}, N_{q}$ and $N_{2}$, and $N_{1}$ and $N_{2}$ is smaller than 0.20 , indicating that the relation was very weak and that the variance of one variable was unlikely to explain the variation of the other variable. The $p$ values of the correlation results are shown in Table 2. The
TABle 1: Pearson correlation matrix.

\begin{tabular}{lcccc}
\hline & $T_{\text {dis }}$ & $N_{q}$ & $N_{1}$ & $N_{2}$ \\
\hline$T_{\text {dis }}$ & 1 & - & - & - \\
$N_{q}$ & 0.476 & 1 & - & - \\
$N_{1}$ & 0.515 & 0.065 & 1 & - \\
$N_{2}$ & 0.261 & -0.045 & 0.184 & 1 \\
\hline
\end{tabular}

TABle 2: $P$ values of the correlations.

\begin{tabular}{lcccc}
\hline & $T_{\text {dis }}$ & $N_{q}$ & $N_{1}$ & $N_{2}$ \\
\hline$T_{\text {dis }}$ & 1 & - & - & - \\
$N_{q}$ & $<0.001$ & 1 & - & - \\
$N_{1}$ & $<0.001$ & 0.508 & 1 & - \\
$N_{2}$ & 0.007 & 0.648 & 0.059 & 1 \\
\hline
\end{tabular}

values below 0.05 are statistically significant, meaning it is 95\% certain that the variations in both variables are not unrelated. In other words, variables $N_{q}, N_{1}$, and $N_{2}$ were likely to explain a part of the variance within the observed queue discharge time.

The regression model that fits the data best is captured by the following equation:

$$
T_{\text {dis }}=2.06+4.84 k_{j}+0.32 N_{1}+0.20 N_{2} .
$$

The standardised regression coefficients, which are used to determine the ranking, for $N_{q}, N_{1}$, and $N_{2}$, are, respectively, $0.46,0.44$, and 0.21 . This indicates that the number of waiting cyclists in the queue is the variable that explained most of the variations in the discharge time, closely followed by the number of merging cyclists from direction 1 . The $R$-squared value of regression equation (9) is 0.499 , indicating that about $50 \%$ of the variance in the discharge time was explained by the model.

The regression model for the discharge time shows that the influence of merging cyclists depended on the direction of the merge. The impact of the direction with respect to the 
influence of the jam density was captured by the bicycle equivalent (BE) value. A BE value of 1.31 was found for bicyclists that merged by taking a sharp turn ( $\sim 90$-degree angle, direction 1), and a BE of 0.83 was found for cyclists that overtook from behind (direction 2). This means that merging behaviour from the side disrupted the discharge process and caused a delay to the last cyclist in the original queue, whereas merging behaviour from behind affected the discharge time less. Using the $\mathrm{BE}$ values, the effect of merging cyclists from different directions was recalculated to a uniform expression for merging flow, expressed in bicycle equivalent units. The results are visualised in Figure 6, showing an increase in the queue discharge rate with both increasing jam density and corrected merging flow. The observed queue discharge rate ranged between 1.3 and $3.3 \mathrm{bic} / \mathrm{s}$ over a $3 \mathrm{~m}$ wide cycle path, which translates into 1,500 to $4,200 \mathrm{beu} / \mathrm{h} / \mathrm{m}$. These estimates were valid only during the discharge process itself, which lasted up to 7 seconds in this study. It is unlikely that this high flow can be maintained for a full hour under real traffic conditions, but for comparison reasons, the rates are expressed in an hourly rate. The large variation in the queue discharge rate was explained by different combinations of jam density and merging cyclists. The lower values were related to low jam density values and low merging flow rate, whereas the highest queue discharge values were achieved by a high presence of merging cyclists. The maximum value of $4,200 \mathrm{beu} / \mathrm{h} / \mathrm{m}$ was found at a jam density of $0.4 \mathrm{bic} / \mathrm{m}^{2}$ and merging flow of $1.75 \mathrm{beu} / \mathrm{s}$.

\section{Validation Experiment}

The results of the case study show a positive relation between jam density and queue discharge time, as well as a positive influence of jam density and merging cyclists on the queue discharge rate. These findings are validated in an experimental setting which allows to control the group composition by using the same set of cyclists for each queuing event. By doing so, the variability in the observed queue discharge time, caused by differences between cyclists, e.g., bicycle type and experience, is reduced.

The setup of the experiment was as follows. We asked a group of 20 cyclists to cycle as they would normally do in daily life and to stop when they reached a mimic traffic signal. While approaching the intersection and the signal was red, the cyclists were free to pick their position while forming a queue. An example of such a queuing event is shown in Figure 7(a). Once the sign turned green, the cyclists started cycling again, and the queue cleared. The group of cyclists approached the mimic intersection multiple times, while mixing the ordering every time such that differences in the cycling style would not be dominant. The track did not include a side path, meaning that merging cyclists could only influence the queue discharge process by overtaking. The track width was varied between two and three meters to get different jam density values. Recall that the path in the case study was a two-way cycle path of $3 \mathrm{~m}$ width and that this full width was only used in the dense queues.

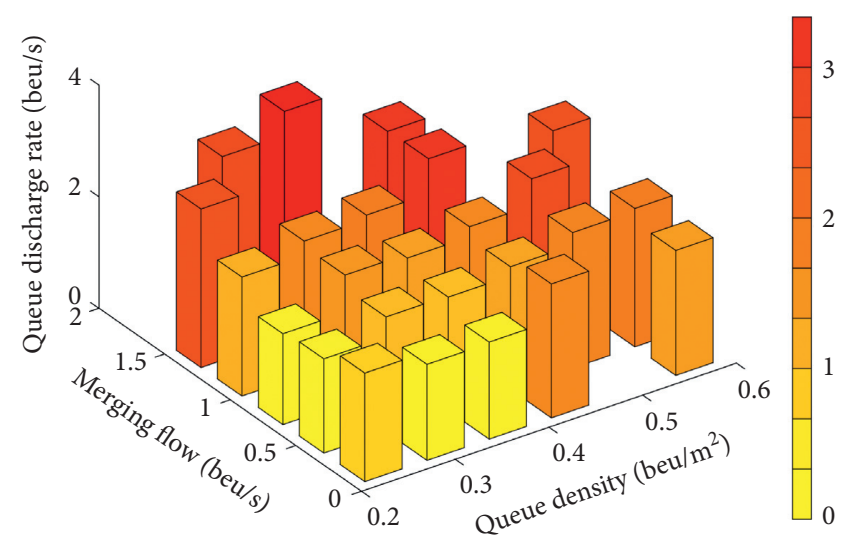

FIgURE 6: Queue discharge rate at different jam densities and merging flows, expressed in bicycle equivalent units.

The group of participants consisted primarily of students from an American university who were in their twenties. A questionnaire learned that about half of the participants use a bike several times per week in their daily life, while a small portion indicated that they never cycle. Nevertheless, they indicated that their cycling confidence during the experiment was good. All cyclists participated on a rental bike in which they had time to become familiar within the days prior to the experiment.

The procedure to retrieve jam density, queue discharge time, and discharge rate was similar to that for the case study. The only difference is the dimension of the waiting area. A fixed queue length of $7 \mathrm{~m}$ was chosen instead of $6.7 \mathrm{~m}$ such that the boundary was clearly distinguishable by an existing cross-line on the path, see Figure $7(\mathrm{~b})$. The total length of the queue was 8 to $15 \mathrm{~m}$ and thus exceeded the length of the waiting area in each run. A total of 7 runs were recorded, which is deemed sufficient to qualitatively validate the positive effect of jam density on the queue discharge rate.

6.1. Experiment Results. The combined jam density and discharge time of the experiment are shown in Figure 8. Two initial observations can be made based on it: first, we find the points further to the right, so the jam density is higher in the experiment than what was observed in the case study. And second, the points are higher up in the graph, so more time is needed to clear the queue, resulting in larger queue discharge times.

The observed discharge times at $k_{\text {jam }}=0.64 \mathrm{bic} / \mathrm{m}^{2}$ are widespread, which can be explained when looking at the discharge process. The queue that took approximately 10 seconds to clear was influenced by a cyclist that had difficulty to start cycling and was overtaken in the process, while the cyclists in the queue that only needed 6 seconds to clear were restarting to cycle so fast that a gap appeared to the cyclists behind them in the queue, indicating that the process was faster than anticipated.

For validation of the relationship, we also show the regression line, which is given by $T_{\text {dis }}=4.63+5.29 k_{\text {jam }}$ and $R$-squared $=0.212$. It shows that in the controlled experiment also, the queue discharge rate increases with jam density. However, the overall flow rate is lower than in the 


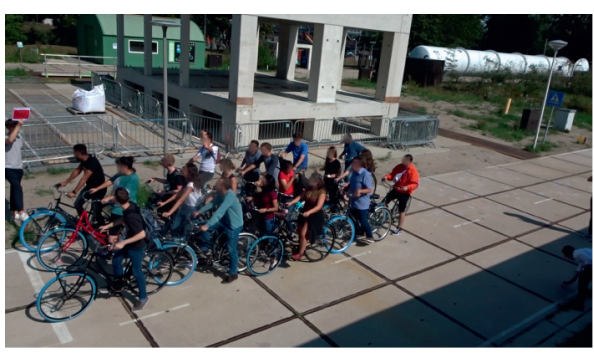

(a)

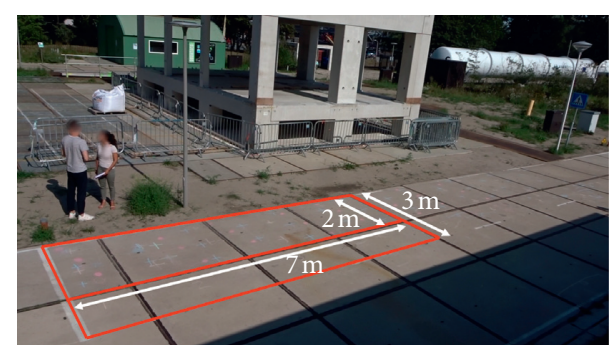

(b)

Figure 7: Example of a queuing event in the experiment (a) and cleared path showing the waiting area indicated with red lines (b).

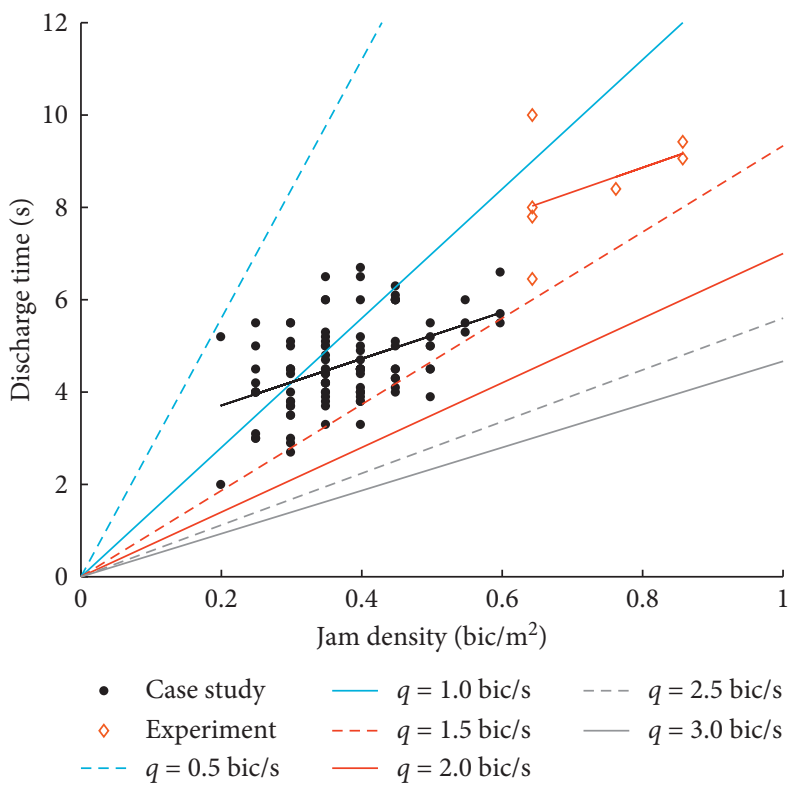

Figure 8: Combined results of the case study and experiment.

case study. In the experiment, the flow rate increases from 1.2 to $1.4 \mathrm{bic} / \mathrm{s}$, while in the case study, a discharge rate of $1.5 \mathrm{bic} / \mathrm{s}$ was reached. Although the number of observations is limited, the discharge rate shows a positive response to an increase in jam density. As expected based on different group compositions, the regression model from Section 5.1 is unable to predict the discharge times for the higher jam density values in the experiment. On the contrary, the experiment results do show that the queue discharge time and rate are indeed positively influenced by the density of the queue.

Merging by overtaking was observed only on two occasions in the experiment. In both runs, there was exactly one cyclist that merged into the original queue and several partial overtaking manoeuvres, which were not completed before passing the stopping line. These two merging events are too limited to validate the method proposed in Section 3.2. Nevertheless, it is interesting to mention that merging by overtaking is also observed in an experimental setting.

\section{Discussion and Future Research}

Both the case study results and the experiment findings show that a positive relation exists between the jam density and both the queue discharge time and the discharge rate for a queue of fixed length.

The trend lines in Figure 8 show that the discharge rates in the case study increase from 0.8 to $1.5 \mathrm{bic} / \mathrm{s}$, and in the experiment, they increase from approximately 1.2 to $1.4 \mathrm{bic} / \mathrm{s}$ for the total width of the path. When extrapolating these flow rates to an hourly saturation rate, the results for the case study roughly increase from 1,000 to $1,800 \mathrm{bic} / \mathrm{h} / \mathrm{m}$, while the experiment give rates between 1,400 and $1,700 \mathrm{bic} / \mathrm{h} / \mathrm{m}$. These results are below the lowest saturation rate of $2,000 \mathrm{bic} / \mathrm{h} / \mathrm{m}$ that was reported in the literature by Seriani et al. [15]. A possible explanation for this could be the absence of hastiness in the experiment.

The analysis of the case study results identified three factors that influenced the time for clearing a queue of fixed length, being the number of cyclists in the queue, merging cyclists from the side, and merging cyclists from behind. Merging behaviour from the side disrupted the discharge process and caused a delay to the last cyclist in the original queue, whereas merging behaviour from behind affected the discharge time less. Unfortunately, the number of observed overtaking manoeuvres in the experiment was too small to validate the impact of merging cyclists, and more research is 
needed before it can be used in practice. Furthermore, it would be interesting to study more locations to find the effect of geometry, population, weather, and other factors in the future research. Possibly, other means of data collection could contribute to this, e.g., GPS-trajectories [24], mobile phone data [25], or drone counts [26].

The queue discharge rate increased with increasing jam density. This indicates that a higher discharge flow can be obtained if cyclists create a denser queue. Further exploring this dependency on jam density would be interesting, e.g., to optimise the throughput at an intersection. Does the positive influence of jam density on the flow rates maintains when cyclists queue up closer together, or is there a maximum? Furthermore, more behavioural research is needed to find the best way to nudge cyclists into queuing up closer together in a real-life situation. Other possible research directions are allowing for a dynamic queue length instead of a fixed length, as well as analysing the exact spatial configuration of the queue to identify the relation to the merging flow. Open spaces in the queue (local density minima) could initiate merging behaviour from different directions. Personal characteristics, i.e., age, gender, and level of fitness, can be included to retrieve a more specific impact factor for merging cyclists, as well as specifying the type of bicycle, i.e., electrical, regular, or cargo.

\section{Conclusion}

This study analysed the queue discharge process of cyclists at a controlled intersection, focusing on how jam density and merging cyclists influence the discharge rate. The jam density was found to positively influence the queue discharge rate. Furthermore, it was found that cyclists who merged by overtaking contributed more to the observed discharge rate compared to cyclists that were standing in the original queue. Cyclists that merged from a direction perpendicular to the queuing direction were found to hinder the discharge process, decreasing the observed discharge rate. The positive relation between jam density and queue discharge time and discharge rate was validated in a controlled experiment.

The results of this study contribute to the development of bicycle models which help in assessing new plans for the bicycle infrastructure layout or measures to minimise delay at intersections.

\section{Data Availability}

The data of the queuing events (cyclist counts and discharge times) that are used in this study are available from the corresponding author upon request.

\section{Disclosure}

Part of this research was presented at the 97th Annual Meeting of the Transportation Research Board (2018, Washington DC, United States). The abstract is available through pure.tudelft.nl.

\section{Conflicts of Interest}

The authors declare that there are no conflicts of interest regarding the publication of this article.

\section{Acknowledgments}

This research was supported by the ALLEGRO project, which was financed by the European Research Council (Grant no. 669792) and the Amsterdam Institute for Advanced Metropolitan Solutions.

\section{References}

[1] E. Heinen, B. Van Wee, and K. Maat, "Commuting by bicycle: an overview of the literature," Transport Reviews, vol. 30, no. 1, pp. 59-96, 2010.

[2] M. Winters, R. Buehler, and T. Götschi, "Policies to promote active travel: evidence from reviews of the literature," Current Environmental Health Reports, vol. 4, no. 3, pp. 278-285, 2017.

[3] J. Pucher and R. Buehler, "Cycling for everyone," Transportation Research Record: Journal of the Transportation Research Board, vol. 2074, no. 1, pp. 58-65, 2008.

[4] D. Ton, D. C. Duives, O. Cats, S. Hoogendoorn-Lanser, and S. P. Hoogendoorn, "Cycling or walking? determinants of mode choice in the Netherlands," Transportation Research Part A: Policy and Practice, vol. 123, pp. 7-23, 2019.

[5] KiM, "Mobiliteitsbeeld 2019," Technical Report, Netherlands Institute for Transport Policy Analysis, Noordwijk, Netherlands, 2019.

[6] H. Botma and H. Papendrecht, "Traffic operation of bicycle traffic," Transportation Research Record: Journal of the Transportation Research Board, vol. 1320, no. 1, pp. 65-72, 1991.

[7] S. Hoogendoorn and W. Daamen, "Bicycle headway modeling and its applications," Transportation Research Record: Journal of the Transportation Research Board, vol. 2587, no. 1, pp. 34-40, 2016.

[8] J. Wang and H. Wei, "Traffic segregation on spatial and temporal bases: the experience of bicycle traffic operations in China," Transportation Research Record: Journal of the Transportation Research Board, vol. 1396, pp. 11-17, 1993.

[9] F. P. D. Navin, "Bicycle traffic flow characteristics: experimental results and comparisons," ITE Journal, vol. 64, no. 3, pp. 31-37, 1994.

[10] J. Zhang, W. Mehner, E. Andresen et al., "Comparative analysis of pedestrian, bicycle and car traffic moving in circuits," Procedia-Social and Behavioral Sciences, vol. 104, pp. 1130-1138, 2013.

[11] X. H. Deng and J. Xu, "Study on bicycle flow characteristics atintersections," in Civil Engineering and Urban Planning III, G. Mohammadian and W. Cicek, Eds., Taylor \& Francis Group, London, UK, pp. 393-399, 2014.

[12] B. Goñi-Ros, Y. Yuan, W. Daamen, and S. P. Hoogendoorn, "Empirical analysis of the macroscopic characteristics of bicycle flow during the queue discharge process at a signalized intersection," Transportation Research Record: Journal of the Transportation Research Board, vol. 2672, no. 36, pp. 51-62, 2018.

[13] Z. Li, M. Ye, Z. Li, and M. Du, "Some operational features in bicycle traffic flow," Transportation Research Record: Journal of the Transportation Research Board, vol. 2520, no. 1, pp. 18-24, 2015. 
[14] X. Chen, K. An, and L. Li, Study on the Influencing Factors of Bicycle Lane Capacity, Transportation Research Board, Washington, DC, USA, 2014.

[15] S. Seriani, R. Fernandez, and E. Hermosilla, "Experimental study for estimating capacity of cycle lanes," Transportation Research Procedia, vol. 8, pp. 192-203, 2015.

[16] S. Jin, X. Qu, D. Zhou, C. Xu, D. Ma, and D. Wang, "Estimating cycleway capacity and bicycle equivalent unit for electric bicycles," Transportation Research Part A: Policy and Practice, vol. 77, pp. 225-248, 2015.

[17] J. Cao, R. Liu, and B. Li, "Research on the bicycle flow in signalized intersections with video-based detection technologies," in Proceedings of the ICTIS 2011, pp. 629-635, Wuhan, China, July 2011.

[18] Y. Yuan, B. Goñi-Ros, M. Poppe, W. Daamen, and S. P. Hoogendoorn, "Analysis of bicycle headway distribution, saturation flow and capacity at a signalized intersection using empirical trajectory data," Transportation Research Record: Journal of the Transportation Research Board, vol. 2673, no. 6, pp. 10-21, 2019.

[19] R. P. Roess and E. S. Prassas, The Highway Capacity Manual: A Conceptual and Research History, Springer, Berlin, Germany, 2014.

[20] N. Y. Cao and K. Sano, "Estimating capacity and motorcycle equivalent units on urban roads in Hanoi, Vietnam," Journal of Transportation Engineering, vol. 138, no. 6, pp. 776-785, 2012.

[21] X. Chen, H. Han, J. Ye, S. Ma, and Y. Xu, "Normalized volume measurement for nonmotorized traffic flow mixed with mopeds," Transportation Research Record: Journal of the Transportation Research Board, vol. 2239, no. 1, pp. 9-15, 2011.

[22] X. Chen, H. Han, and B. Lin, "Developing bicycle equivalents for mopeds in Shanghai, China," Transportation Research Record: Journal of the Transportation Research Board, vol. 2317, no. 1, pp. 60-67, 2012.

[23] Transportation Research Board, Highway Capacity Manual, National Research Council, Washington, DC, USA, 2000.

[24] K. Christian, S.-H. Cho, S.-Y. Kho, and D.-K. Kim, "Bayesian models with spatial autocorrelation for bike sharing ridership variability based on revealed preference GPS trajectory data," IET Intelligent Transport Systems, vol. 13, no. 11, pp. 16581667, 2019.

[25] M. Ghahramani, M. Zhou, and G. Wang, "Urban sensing based on mobile phone data: approaches, applications, and challenges," IEEE/CAA Journal of Automatica Sinica, vol. 7, no. 3, pp. 627-637, 2020.

[26] D. Kim, "Pedestrian and bicycle volume data collection using drone technology," Journal of Urban Technology, vol. 27, no. 2, pp. $45-60,2020$. 